\title{
Cosmological constant problem: deflation during inflation
}

\author{
Felipe Canales, ${ }^{1}$ Benjamin Koch, ${ }^{1,2}$ Cristobal Laporte, ${ }^{1}$ and Ángel Rincón ${ }^{3}$ \\ ${ }^{1}$ Pontificia Universidad Católica de Chile Instituto de Física, \\ Pontificia Universidad Católica de Chile, Casilla 306, Santiago, Chile \\ ${ }^{2}$ Institut für Theoretische Physik, Technische Universität Wien, \\ Wiedner Hauptstrasse 8-10, A-1040 Vienna, Austria \\ ${ }^{3}$ Instituto de Física, Pontificia Universidad Católica de Valparaíso, \\ Avenida Brasil 2950, Casilla 4059, Valparaso, Chile ${ }^{*}$
}

\begin{abstract}
We argue that the discrepancy between the Planck mass scale and the observed value of the cosmological constant can be largely attenuated if those quantities are understood as a result of effective, and thus scale-dependent, couplings. We exemplify this mechanism for the early inflationary epoch of the universe by solving the corresponding effective gap equations, subject to an energy condition. Several non-trivial checks and extensions are discussed. A comparison of our results to the renormalization group flow, obtained within the asymptotic safety program reveals a stunning agreement.
\end{abstract}

\section{Contents}

\section{Introduction}

A. Classical $\Lambda$ dominated universe

B. The cosmological constant problem in the very early universe

\section{2}

II. Scale-Dependence in Gravity
A. Scale setting
B. Modified Friedmann equations
C. Null energy condition (NEC)

\section{Results and discussion}

A. Equation of state of scale-dependent $G(t)$

B. Deflation of the cosmological constant problem

C. Comparison with the functional renormalization group

\section{Conclusions}

\section{References}

\section{INTRODUCTION}

The cosmological constant problem (CCP) seems to point towards deep misconceptions in our current understanding of the interplay between quantum field theory and general relativity [1]. In this article, we want to point out that one possible misconception is that, one makes reference to gravitational couplings at different epochs of the universe, in particular for the most dramatic version of the CCP. This reference to different epochs of the Universe is however inconsistent if there is an epoch where the gravitational couplings are not constant, as it is expected for example from quantum effects

*Electronic address: angel.rincon@pucv.cl which are can induce an effective running of the fundamental couplings (e.g. Newtons coupling $G_{0} \rightarrow G_{k}$ ). It is clear that for quantum effects to be notable one needs a violent epoch of the universe. Due to our current understanding the early inflationary period [4, 5] is the best candidate for such non-classical corrections to be relevant. In particular, during inflation the quantum effects and thus the scale-dependence of effective couplings can be expected to be much stronger than during other later evolution periods. There are two arguments that support this idea. First, since the very early universe is typically related to Planck-time and Planck-distances it is natural to expect important quantum gravity corrections [6 13, such as scale-dependence of the effective couplings. Second, in these very early times, other scales such as particle masses, which might moderate the scale-dependence are still irrelevant. During later times, to the contrary, there are no Planck distances involved and there are also logarithmically running particle masses contributing which could diminish the scale-dependence of the gravitational couplings further. Thus, it makes sense to focus this type of study on the first inflationary period of the Universe. Under such extreme conditions the gravitational couplings including the cosmological "constant" can not be expected to be constant [16] and thus one has to take care when realizing a comparison of those couplings at different times.

In the following sections we will elaborate a scaledependent vacuum dominated model which incorporates the following features, which are in agreement with the ideas mentioned above: i) scale-dependence within effective gap equations ii) inflationary behavior of the cosmological scale factor $a(t)$ iii) non-trivial evolution of the vacuum energy density and the gravitational coupling and iv) exponential suppression of the CCP during the inflationary period. This is achieved by applying methods that have been successfully used in order to include quantum correction into gravitational theories, black hole backgrounds, wormholes, and particular cosmological problems [17 55]. 


\section{A. Classical $\Lambda$ dominated universe}

Based on the cosmological principle and assuming a flat Universe, one uses the line element

$$
\mathrm{d} s^{2}=-\mathrm{d} t^{2}+a(t)^{2}\left[\mathrm{~d} r^{2}+r^{2} \mathrm{~d} \Omega_{2}^{2}\right]
$$

as ansatz for the metric field, where $\mathrm{d} \Omega_{2}^{2}=\mathrm{d} \theta^{2}+$ $\sin ^{2}(\theta) \mathrm{d} \phi^{2}$. The scale factor gives

$$
a(t)=a_{i} \mathrm{e}^{t / \tau}
$$

where

$$
\tau= \pm \sqrt{\frac{3}{\Lambda_{0}}}
$$

We will only use the positive sign in (3), which corresponds to an expanding universe.

\section{B. The cosmological constant problem in the very early universe}

There are different versions of the CCP to be found in the literature. Most of them can be cast in the dimensionless ratio between the highest UV cut-off $M_{U V}$ of the quantum field theory one is using to calculate the vacuum energy density and the energy density $\rho=\Lambda_{0} / G$ observed due to the accelerated expansion of the universe [3]

$$
r \approx \frac{M_{U V}^{4}}{\rho} \approx \frac{M_{U V}^{4} G}{\Lambda_{0}}
$$

Figure 1 shows the exceedingly large values of (4) for various emblematic energy scales $M_{U V}$ from neutrino physics, proton mass, top mass, up to the Planck scale. For a good theoretical prediction, the value of (4) is supposed to be of order one, as indicated by the orange line. Clearly, there is a dramatic discrepancy.

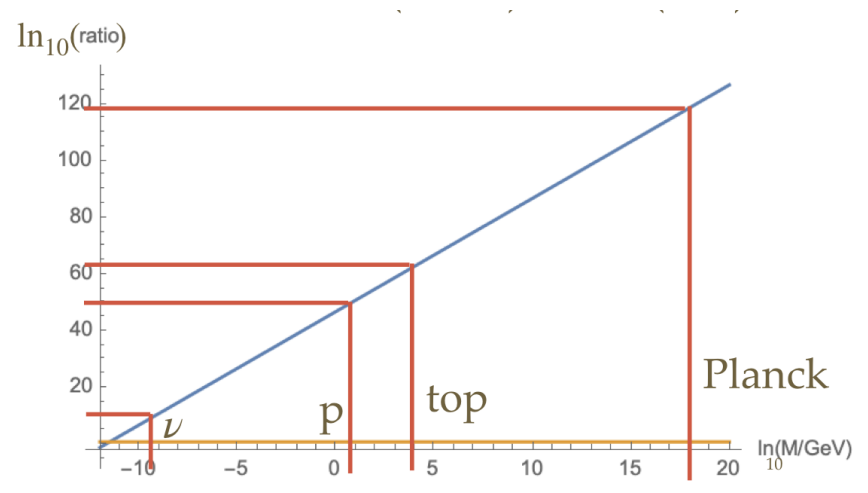

FIG. 1: The blue line indicates the logarithm of the dimensionless ratio (4) as a function of a cut-off mass scale $M_{U V}$. A good agreement between the theoretical estimate and the observational value is indicated by the orange line.
As explained in the introduction, in this paper we will focus on the early expansion of the universe. During this epoch, governed by pure gravity, the only dimensionful quantity is actually the Planck mass given by $m_{P}^{-1}=\sqrt{8 \pi G}$. If one uses this scale as UV scale $M_{U V}^{2} \approx 1 / G$ to estimate the vacuum energy density, the ratio (4) simplifies to

$$
r_{i n f l} \approx \frac{1}{G \cdot \Lambda_{0}} \approx 10^{122}
$$

which is the line labeled with "Planck" in figure 1. The fact that (5) differs from one by more than one hundred orders of magnitude is known as the cosmological constant problem in its worst form. There have been numerous attempts to either solve, alleviate, or to deny this problem [12, 56 66, but up to now the quarrel continues and no agreement has been achieved.

Since our purely gravitational approach ceases to be valid as soon as further mass scales such as the top mass appear, we only can attempt to explain the discrepancy of 66 orders of magnitude spanned by the value of the ratio (5) between the top mass and the Planck mass.

\section{SCALE-DEPENDENCE IN GRAVITY}

A very powerful tool in quantum field theory is the use of effective actions, which incorporate quantum effects in a scale-dependent modification of the classical action. This type of scenario has been proposed and studied in the context of gravity in different ways and with different methods 67 76]. In the so-called Einstein-Hilbert truncation the effective action reads

$$
\Gamma\left[g_{\mu \nu}, k\right]=\int \mathrm{d}^{4} x \sqrt{-g}\left[\frac{1}{16 \pi G_{k}}\left(R-2 \Lambda_{k}\right)+\mathcal{L}_{M}\right],
$$

where $\Lambda_{k}$ and $G_{k}$ are the cosmological and Newton scaledependent couplings, respectively. A great advantage of working with effective quantum actions like (6) is that they already incorporate the effects of quantum fluctuations like those being responsible for the CCP. Thus, if the problem is properly addressed in terms of a background solution of this action, no additional quantum corrections need to added. In order to obtain background solutions for this effective action one has to derive the corresponding gap equations. Thus, varying the effective action respect to the inverse metric field, one obtains the corresponding Einstein field equations [73]

$$
G_{\mu \nu}=T_{\mu \nu}^{\mathrm{effec}}=-\Lambda(t) g_{\mu \nu}-\Delta t_{\mu \nu}
$$

where

$$
\Delta t_{\mu \nu}=G(t)\left[g_{\mu \nu} \nabla^{\alpha} \nabla_{\alpha}-\nabla_{\mu} \nabla_{\nu}\right] G(t)^{-1} .
$$

In order to get physical information out of those equations one has to set the renormalization scale in terms of the physical variables of the system under consideration $k \rightarrow k(x, \ldots)$. 


\section{A. Scale setting}

For most types of scale settings [71, 74, 77- 100, the identification $k \rightarrow k(x, \ldots)$ breaks the reparametrization symmetry (7). There are, however, approaches which allow to maintain the symmetries and thus the consistency of the system. We will invoke a procedure which invokes the variational principle for $k$

$$
\frac{\delta \Gamma_{k}}{\delta k}=0
$$

This approach has been proposed in [101 103] and it is a straightforward implementation of background scale independence [104 111]. Relation (9) is not a fixed point condition but rather a scale setting condition, it even can be used when the renormalization group flow has already approached its fixed point 112 .

Further, for highly symmetric systems with a small number of couplings one can attempt to solve (7) for $g_{\mu \nu}(x), G(x)$, and $\Lambda(x)$ directly by the use of a special ansatz [39, 40, 42, 46, 48, 50, 52, 113, motivated by the structure of the classical solution [114], or by imposing an energy condition 113 .

In this paper we will apply both strategies, the variational scale setting (9) and the energy condition mentioned above.

\section{B. Modified Friedmann equations}

Within the ansatz (1) the dynamical variable $a=a(t)$ does only depend on time and not on spatial directions, the same will be assumed for the two additional variables $G=G(t)$ and $\Lambda=\Lambda(t)$. With this, the gap equations (7) take the form of generalized Friedmann equations

$$
\begin{aligned}
\left(\frac{\dot{a}}{a}\right)^{2}-\frac{\Lambda}{3} & =\frac{8 \pi G}{3} \rho(t), \\
2 \frac{\ddot{a}}{a}+\left(\frac{\dot{a}}{a}\right)^{2}-\Lambda & =-(8 \pi G) p(t),
\end{aligned}
$$

where an effective density $(\rho)$ and pressure $(p)$, given by the scale-dependent framework have been identified with the density and pressure parameters of a perfect fluid

$$
\begin{aligned}
\frac{8 \pi G}{3} \rho(t) & \equiv\left(\frac{\dot{a}}{a}\right)\left(\frac{\dot{G}}{G}\right) \\
-(8 \pi G) p(t) & \equiv-2\left(\frac{\dot{G}}{G}\right)^{2}+\left(\frac{\ddot{G}}{G}\right)+2\left(\frac{\dot{a}}{a}\right)\left(\frac{\dot{G}}{G}\right) .
\end{aligned}
$$

Further, one has the scale setting relation 9

$$
\frac{d}{d t}\left[\frac{1}{16 \pi G(t)}(R-2 \Lambda(t))\right]=0 .
$$

There are three equations, namely the two cosmological gap equations 10,11 and the scale setting condition (14). There are also three unknown functions $(a(t), G(t), \Lambda(t))$. However, the system respects general covariance, which implies that $\nabla_{\mu} G^{\mu \nu}=0$ and thus one of the three equations 10, 11, 14 is just a consequence of the other two. This means that one needs one additional relation or condition in order to determine a solution. In the next subsection, we will invoke an energy condition in order to provide the missing equation.

\section{Null energy condition (NEC)}

In an empty universe, a light-like signal traveling on a null geodesic with velocity vector $\ell^{\mu}=d x^{\mu} / d t$ should not perceive any energy density of the background. This should be true for other vacuum contributions to the effective stress energy tensor, such as $\Delta t_{\mu \nu}$, introduced by the scale-dependence of the gravitational coupling

$$
T_{\mu \nu}^{\mathrm{effec}} \ell^{\mu} \ell^{\nu}=-\Delta t_{\mu \nu} \ell^{\mu} \ell^{\nu}=0 .
$$

This is reminiscent of the null energy condition [115]. The above relation is the scale setting condition which we will impose in addition to the cosmological gap equations 10 . 11). The vector field $\ell^{\mu}$ satisfying the geodesic equation can be written as $\ell^{\mu}=C_{0} a^{-1}\left(1,\left(1-\kappa r^{2}\right)^{1 / 2} a^{-1}, 0,0\right)$. Replacing this in 15 gives the NEC condition for the cosmological model

$$
-2\left(\frac{\dot{G}}{G}\right)^{2}+\left(\frac{\ddot{G}}{G}\right)-\left(\frac{\dot{a}}{a}\right)\left(\frac{\dot{G}}{G}\right)=0 .
$$

\section{RESULTS AND DISCUSSION}

The solution of 10, , 11), and $(16)$ that describes an expanding universe is

$$
\begin{aligned}
a(t) & =a_{i} \mathrm{e}^{\frac{t}{\tau}} \\
G(t) & =G_{0}\left[1+\tilde{\xi} \int_{t_{0}}^{t} a\left(t^{\prime}\right) d t^{\prime}\right]^{-1}=\frac{G_{0}}{1+\xi a(t)} \\
\Lambda(t) & =\Lambda_{0}\left[\frac{1+2 \xi a(t)}{1+\xi a(t)}\right]
\end{aligned}
$$

It is direct to see that (17) takes the same form as the classical counterpart (2). A generalization to non zero values of $\kappa$ is straight forward (see version one of [116]). The solution involves four integration constants which we have labeled $\left(a_{i}, G_{0}, \Lambda_{0}, \xi\right)$. They are set by the conditions

$$
\lim _{t \rightarrow 0} a(t)=a_{i}, \quad \lim _{\xi \rightarrow 0} G(t)=G_{0}, \quad \lim _{\xi \rightarrow 0} \Lambda(t)=\Lambda_{0} \cdot(20)
$$

One notes that the parameter $\xi$ plays the role of a control parameter, When setting $\xi$ to zero, the time-dependent 
couplings $G(t)$ and $\Lambda(t)$ are reduced to their constant and thus "classical" counterparts $\left(G_{0}\right.$ and $\left.\Lambda_{0}\right)$. Please note that the expansion rate is given by $\tau= \pm \sqrt{3 / \Lambda_{0}}$, which looks exactly like the classical rate $(3)$. There is however an important conceptual difference since now, $\Lambda_{0}$ is one of the integration constants, while in (3) it was given as input parameter.

\section{A. Equation of state of scale-dependent $G(t)$}

Is there a meaningful equation of state $p=\omega \rho$ for the effective type of pressure and energy density? Subtracting (11) from $3 \times 10$, one finds for the solution (17, 18)

$$
p(t)+\rho(t)=0
$$

Thus, the effective parameters take the form of a "classical" Dark Energy equation of state with $\omega=-1$. Thus, the effective pressure and the effective energy density cancel each other, which explains why the scaledependence parameter $\xi$ does not appear in the solution for $a(t)$.

\section{B. Deflation of the cosmological constant problem}

As explained in the introduction, the CCP in a Planck era universe can be parametrized by the value of $\Lambda \cdot G$ (5). However, the quantum gravity calculation makes reference to the very early Planck era $t_{i}$, while the measurement makes reference to the more recent history of the universe, long after the end of inflation $t_{f}$. scaledependence can offer alleviation of this problem since it allows for a continuous evolution of $\Lambda(t) \cdot G(t)$ from the Planck era $t_{i}$ to the end of inflation $t_{f}$ as shown in the left panel of figure 3. As argued in the introduction, we assume that the most dominant scaling effects occur in this period and that in later times, after $t_{f}$, the scaling effects will be dominated by coupling to external particle scales $m_{i}$, which only run logarithmically.

In order to show that the CCP can be addressed effectively within our framework, we impose six conditions on the scale-dependent solution 17,19 during inflation. One has to check whether those conditions can be met by a choice of the constants $t_{i}, t_{f}, G_{0}, \Lambda_{0}, a_{i}, \xi$.

a) At the initial time $t_{i} \equiv 0$ one demands

$$
a\left(t_{i}\right)=1 \text {. }
$$

and

$$
\Lambda\left(t_{i}\right) \cdot G\left(t_{i}\right) \approx 1,
$$

which meets the theoretical expectation.

b) At the end of inflation $t_{f}$ one imposes that the gravitational coupling has evolved to a value close to the current measured value

$$
G\left(t_{f}\right) \approx G_{N}
$$

and that the cosmological constant problem has gravitationally evolved to

$$
\Lambda\left(t_{f}\right) \cdot G\left(t_{f}\right) \approx 5 \cdot 10^{-66}
$$

around the magnitude dictated by the heaviest standard model particle.

c) The observed flatness of the universe suggest further that during this epoch (from $t_{i}$ to $t_{f}$ ) of rapid growth, the universe expanded at least sixty efolds 117

$$
N_{e} \geq 60
$$

By imposing the conditions $22,23,24$ one finds

$$
\begin{aligned}
a_{i} & =1 \\
t_{f} & =N_{e} \tau \\
\Lambda_{0} & =\frac{(1+\xi)^{2}}{G_{N}(1+2 \xi)\left(1+\xi e^{N_{e}}\right)}
\end{aligned}
$$

In order to analyze the condition (25), we put it into an order of magnitude inequality, accepting five orders of magnitude deviation from the value given in 25

$$
10^{-71} \lesssim \Lambda\left(t_{f}\right) \cdot G\left(t_{f}\right) \lesssim 10^{-61}
$$

This double inequality puts conditions on the two remaining constants $N_{e}$ and $\xi$. The region of allowed values for those two constants, according to $(30)$, is shown in the blue contour in figure 2 , where we have defined $\xi=e^{\xi_{V}}$. From this figure one notes that the condition 30 never

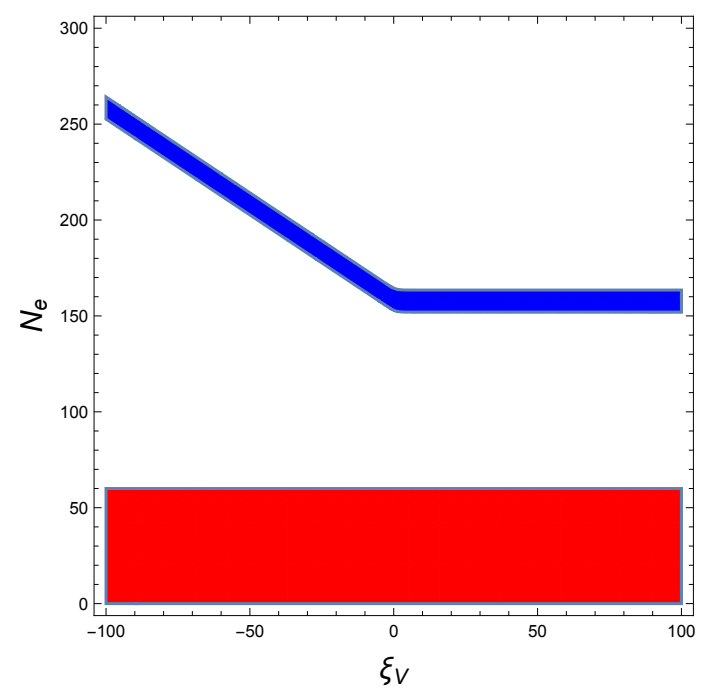

FIG. 2: $N_{e}, \xi_{V}$ parameter space, where the blue contour is fulfilling both conditions of 30 and the red colour is excluded from the observational condition 26 .

gets in conflict with the observational bound (26). It is thus shown that substantial alleviation of the cosmological constant problem within the presented framework 
can be achieved. For this one does need the inflationary period to last at least $\ln \left(10^{66}\right) \approx 150$ e-folds. This is significantly larger than the experimental lower bound, but it does not cause tension with observational data since it is very hard to establish a distinctive upper bound for this quantity [118.

The scale-dependent evolution could be incorporated into more realistic models of inflation [119], but this goes beyond the scope of this article. It is further tempting to speculate whether the suppression of $G(t)$ in the first equality of 18 can be used in combination with the scale-dependence of other couplings in the postinflationary universe 120 129] in order to contribute to the solution of the hierarchy problem [130 132]. This possibility will be explored in future studies.

\section{Comparison with the functional renormalization group}

Even though our calculation never makes use of specific quantum gravity beta functions, it is tempting to make a comparison with the findings in such an approach. The discussion below will be complementary to the findings in [12, 13, where strong effects of the evolution of couplings on the cosmological constant problem have been pointed out in context with a possible infra-red instability of asymptotically save gravity [14, 15.

Using functional renormalization group (RG) methods, in particular, the Wetterich equation [69, 70, 72, it is possible to obtain a prediction for the scale-dependent couplings in gravitation actions such as (6). The dimensionless couplings $\hat{g}(k)=\hat{G}(k) k^{2}$ and $\hat{\lambda}(k)=\hat{\Lambda}(k) / k^{2}$ are found for the dimensionless renormalization scale $\hat{t}=\log \left(k / k_{0}\right)$

$$
\begin{aligned}
\hat{g}(\hat{t}) & =\frac{g_{0} e^{2 \hat{t}}}{1+g_{0}\left(e^{2 \hat{t}}-1\right) / g^{*}}, \\
\hat{\lambda}(\hat{t}) & =\frac{g^{*} \lambda_{0}+e^{-2 \hat{t}}\left(e^{4 \hat{t}}-1\right) g_{0} \lambda^{*}}{1+g_{0}\left(e^{2 \hat{t}}-1\right) / g^{*}},
\end{aligned}
$$

where $g^{*}$ and $\lambda^{*}$ are the values of the ultra violet fixed point predicted by the Asymptotic Safety (AS) conjecture [67, 76] and found within the functional RG approach [133 135. Further, $\lambda_{0}$ and $g_{0}$ are initial values defined at an intermediate renormalization scale $\hat{t}=0$. For the CCP in the very early universe the relevant quantity is the product of the dimensionful couplings $\hat{G}(\hat{t}) \cdot \hat{\Lambda}(\hat{t})$. In four dimensions the product of the dimensionful couplings is equal to the product of the dimensionless couplings 31,32

$$
\hat{G}(\hat{t}) \hat{\Lambda}(\hat{t})=g(\hat{t}) \lambda(\hat{t}) .
$$

Therefore, a comparison of the naturally dimensionless product 33 allows for much more robust statements than a comparison of the individual dimensionful or dimensionless couplings.
The one million dollar question is whether there is a scale setting $\hat{t}=\hat{t}(t)$ for which the product 33 obtained from AS agrees, at least qualitatively, with the product $G(t) \cdot \Lambda(t)$ derived in our scale-dependent cosmological solution 18, 19p. In the ultraviolet (UV) and close to the separatrix [136] of the RG flow one can approximate 31 and 32 for $e^{2 \hat{t}}, g^{*} / g_{0} \gg 1$, which gives

$$
\hat{g}(\hat{t}) \hat{\lambda}(\hat{t})=g^{*} \lambda^{*}\left(\frac{g^{*} \lambda_{0}}{g_{0} \lambda^{*}}+e^{2 \hat{t}}\right)\left(e^{2 \hat{t}}+\frac{g^{*}}{g_{0}}\right)^{-2} .
$$

By inspection one realizes that the simple replacements

$$
g^{*} \lambda^{*} \rightarrow G_{0} \Lambda_{0}, \quad g_{0} \rightarrow \frac{G_{0}}{a_{i} \xi}, \quad \hat{t} \rightarrow-\frac{t}{2 \tau},
$$

together with the choice $\lambda_{0}=2 \lambda^{*}$, leads to a stunning exact matching

$$
\left.\hat{g}(\hat{t}) \hat{\lambda}(\hat{t})\right|_{(3.19)}=G(t) \Lambda(t) .
$$

This means that for a proper scale setting and a proper choice of the flow trajectory, both approaches show the same type of deflation of the cosmological constant problem. Graphically this behavior is shown in figure 3 .
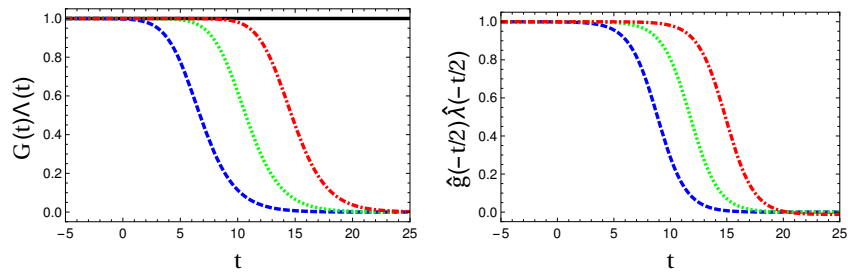

FIG. 3: LEFT: Evolution of $G(t) \cdot \Lambda(t)$ for $\xi=0$ (solid black line), $\xi=0.001$ (dotted-dashed red line), $\xi=0.01$ (dotted green line) and $\xi=0.1$ (dashed blue line). The rest of the parameters have been taken to be unity. RIGHT: Evolution of $\hat{\lambda}(-t / 2) \cdot \hat{g}(-t / 2)$ as given by AS for $g_{0}=0.001$ (dashed blue line), for $g_{0}=0.01$ (dotted green line), and for $g_{0}=0.1$ (dot-dashed red line). Other constants were set to unity.

Comparing our solutions to the AS predictions, on one hand, we observe that the behavior of the product $G \cdot \Lambda$ as a function of $t$ is exemplified in the figure (3) (LEFT). One notes that $G(t) \cdot \Lambda(t)$ experiences a strong suppression. In addition, for smaller values of $\xi$ the evolution of the couplings starts at later times. Apart from this change, the functions are form-invariant under $t \rightarrow t-t_{i}$ transformations. This means that the value of $\xi$ can be reabsorbed in a shift in the initial or final conditions for $a(t), G(t)$, and $\Lambda(t)$. On the other hand, the same features are shown in (3) (RIGHT).

Clearly, this agreement can still be a coincidence but it appears sufficiently interesting to us to mention it here in the discussion. There are several comments about the result (36) and the identifications 35 to be made. The result (36) is not the product of a very particular cutoff identification. It is straightforward to check that the 
same agreement can be obtained from different beta functions arising from different cut-off prescriptions. From (35) one sees from the UV/AS perspective that the control parameter $\xi$ is not necessarily small, but a rather large number. This means that scale-dependence in this regime of high energies is a dominant effect and not just a small correction to classical behavior. One further sees from (35) that the product $\Lambda \cdot G$ in the UV is predicted to be just the product of the two fixed points $g^{*} \cdot \lambda^{*}$ of the RG flow. The values of those fixed points are typically found to be of order $\sim 0.2$, the product is thus not dramatically far from unity, just as we had supposed in our working assumptions. Finally, one notes from (35) that the scale identification is in agreement with our intuitive expectations, since it associates very early times $t \rightarrow-\infty$ to very large RG scales $k \rightarrow \infty$ and late times $t \rightarrow \infty$ to small RG scale $k \rightarrow 0$.

We can not resist to insist that the identity (36) is absolutely not trivial. It links two completely different and independent calculations. One is implementing quantum effects into a gravitational theory by means of effective quantum field theory and the other solving the modified Friedmann equations (10, 11) subject to a simple energy condition (16). The support of both approaches is mutual. One can read the agreement of 18,19 with 31 . 32 as support of the approach and the solution presented in this paper. One can also conclude that AS is in good shape to contribute to the solution of the CCP, if one chooses a flow line which is in sufficiently close vicinity of the separatrix line $\left(g_{0} \ll 1\right)$.

\section{CONCLUSIONS}

In this article, we presented a study on the early inflationary universe in the context of scale-dependence. We give reasons, why it can be expected that the lead- ing gravitational couplings experience scale-, in particular time-dependence in this early epoch. It is then argued that, if one accepts possible scale-dependence of the couplings, then the CCP is ill-defined since it makes an identification of the product $\Lambda(t) \cdot G(t)$ at two different times of the cosmological evolution. We conjecture that this misconception and thus the CCP can be resolved if one takes into account a proper scale-dependence of those couplings.

As a concrete example, we solve the gap equations (7) of the scale-dependent gravitational action in the Einstein-Hilbert truncation for the homogenous isotropic line element (1) subject to a null energy condition (15). It is found that within this solution $(17,19)$ the scale factor $a(t)$ grows exponentially. Further, the couplings $\Lambda(t)$ and $G(t)$ have the expected strong time-dependence. It is shown that the time evolution of $\Lambda(t) \cdot G(t)$ indeed allows accommodating a substantial alleviation of the CCP in agreement with the conditions $(22,25)$ and the observational lower bound (26).

We note two particular features of our results. First, the contribution of the scale-dependent coupling $G(t)$, can be written as an ideal fluid obeying an equation of state, which is usually associated with vacuum energy density. This is a strong support for the chosen energy condition (15), because it shows that the condition guaranties a vacuum type equation of state for the scale dependent contribution $\Delta t_{\mu \nu}$ of the stress energy tensor. Second, a comparison between the product $\Lambda(t) \cdot G(t)$, deduced in this paper, with the product $\hat{\lambda}(\hat{t}) \cdot \hat{g}(\hat{t})$, obtained from functional RG methods within the AS scenario, reveals highly non-trivial similarities.

Acknowlegements. B. K. was supported by the Fondecyt 1161150 and Fondecyt 1181694. Á. R. acknowldeges DI-VRIEA for financial support through Proyecto Postdoctorado 2019 VRIEA-PUCV.
[1] R. J. Adler, B. Casey and O. C. Jacob, Am. J. Phys. 63, 620 (1995). doi:10.1119/1.17850

[2] H. Martel, P. R. Shapiro and S. Weinberg, Astrophys. J. 492, 29 (1998) doi:10.1086/305016 astro-ph/9701099.

[3] S. Weinberg, Rev. Mod. Phys. 61, 1 (1989). doi:10.1103/RevModPhys.61.1

[4] A. H. Guth, Phys. Rev. D 23, 347 (1981) [Adv. Ser. Astrophys. Cosmol. 3, 139 (1987)]. doi:10.1103/PhysRevD.23.347

[5] A. D. Linde, Phys. Lett. 108B, 389 (1982) [Adv. Ser. Astrophys. Cosmol. 3, 149 (1987)]. doi:10.1016/03702693(82)91219-9

[6] V. F. Mukhanov, H. A. Feldman and R. H. Brandenberger, Phys. Rept. 215, 203 (1992). doi:10.1016/03701573(92)90044-Z

[7] M. Gasperini and G. Veneziano, Astropart. Phys. 1, 317 (1993) doi:10.1016/0927-6505(93)90017-8 hepth/9211021.

[8] M. Novello, J. Barcelos-Neto and J. M. Salim,
Class. Quant. Grav. 19, 3107 (2002) doi:10.1088/02649381/19/11/323 hep-th/0202169.

[9] A. Ashtekar and J. Lewandowski, Class. Quant. Grav. 21, R53 (2004) doi:10.1088/0264-9381/21/15/R01 grqc/0404018.

[10] T. Thiemann, Lect. Notes Phys. 631, 41 (2003) doi:10.1007/978-3-540-45230-0 3 gr-qc/0210094.

[11] C. Rovelli, PoS QGQGS 2011, 003 (2011) doi:10.22323/1.140.0003 arXiv:1102.3660 [gr-qc]].

[12] C. Wetterich, Phys. Lett. B 773, 6 (2017) doi:10.1016/j.physletb.2017.08.002 arXiv:1704.08040 $[\mathrm{gr}-\mathrm{qc}]]$.

[13] J. Rubio and C. Wetterich, Phys. Rev. D 96, no. 6, 063509 (2017) doi:10.1103/PhysRevD.96.063509 arXiv:1705.00552 [gr-qc]].

[14] W. B. Houthoff, A. Kurov and F. Saueressig, Eur. Phys. J. C 77, 491 (2017) doi:10.1140/epjc/s10052-017-5046-8 arXiv:1705.01848 [hep-th]].

[15] L. Bosma, B. Knorr and F. Saueressig, arXiv:1904.04845 
[hep-th].

[16] N. Afshordi and E. Nelson, Phys. Rev. D 93, no. 8, 083505 (2016) doi:10.1103/PhysRevD.93.083505 arXiv:1504.00012 [hep-th]].

[17] A. Bonanno and M. Reuter, Phys. Rev. D 65, 043508 (2002) doi:10.1103/PhysRevD.65.043508 hepth/0106133.

[18] M. Reuter and H. Weyer, Phys. Rev. D 69, 104022 (2004) doi:10.1103/PhysRevD.69.104022 hepth/0311196.

[19] M. Reuter and H. Weyer, JCAP 0412, 001 (2004) doi:10.1088/1475-7516/2004/12/001 hep-th/0410119.

[20] S. Weinberg, Phys. Rev. D 81, 083535 (2010) doi:10.1103/PhysRevD.81.083535 arXiv:0911.3165 [hep-th]].

[21] S.-H. H. Tye and J. Xu, Phys. Rev. D 82, 127302 (2010) doi:10.1103/PhysRevD.82.127302 arXiv:1008.4787 [hep-th]].

[22] A. Bonanno, A. Contillo and R. Percacci, Class. Quant. Grav. 28, 145026 (2011) doi:10.1088/02649381/28/14/145026 arXiv:1006.0192 [gr-qc]].

[23] A. Eichhorn and H. Gies, Phys. Rev. D 81, $104010 \quad$ (2010) doi:10.1103/PhysRevD.81.104010 arXiv:1001.5033 [hep-th]].

[24] D. F. Litim, Phil. Trans. Roy. Soc. Lond. A 369, 2759 (2011) doi:10.1098/rsta.2011.0103 arXiv:1102.4624 [hep-th]].

[25] J. Grande, J. Sola, S. Basilakos and M. Plionis, JCAP 1108, 007 (2011) doi:10.1088/1475-7516/2011/08/007 arXiv:1103.4632 [astro-ph.CO]].

[26] D. Benedetti and F. Caravelli, JHEP 1206, 017 (2012) Erratum: [JHEP 1210, 157 (2012)] doi:10.1007/JHEP06(2012)017, 10.1007/JHEP10(2012)157 arXiv:1204.3541 [hepth]].

[27] M. Hindmarsh and I. D. Saltas, Phys. Rev. D 86, 064029 (2012) doi:10.1103/PhysRevD.86.064029 arXiv:1203.3957 [gr-qc]].

[28] A. Eichhorn, Phys. Rev. D 86, 105021 (2012) doi:10.1103/PhysRevD.86.105021 arXiv:1204.0965 [grqc]].

[29] P. Doná, A. Eichhorn and R. Percacci, Phys. Rev. D 89, no. 8, 084035 (2014) doi:10.1103/PhysRevD.89.084035 arXiv:1311.2898 [hep-th]].

[30] E. J. Copeland, C. Rahmede and I. D. Saltas, Phys. Rev. D 91, no. 10, 103530 (2015) doi:10.1103/PhysRevD.91.103530 arXiv:1311.0881 [gr-qc]].

[31] A. Bonanno and A. Platania, Phys. Lett. B 750, 638 (2015) doi:10.1016/j.physletb.2015.10.005 arXiv:1507.03375 [gr-qc]].

[32] D. C. Rodrigues, B. Chauvineau and O. F. Piattella, JCAP 1509, no. 09, 009 (2015) doi:10.1088/14757516/2015/09/009 arXiv:1504.05119 [gr-qc]].

[33] L. Modesto and I. L. Shapiro, Phys. Lett. B $\mathbf{7 5 5}, \quad 279$ (2016) doi:10.1016/j.physletb.2016.02.021 arXiv:1512.07600 [hep-th]].

[34] D. C. Rodrigues, B. Koch, O. F. Piattella and I. L. Shapiro, AIP Conf. Proc. 1647, 57 (2015). doi: $10.1063 / 1.4913338$

[35] D. C. Rodrigues, S. Mauro and Á. O. F. de Almeida, Phys. Rev. D 94, no. 8, 084036 (2016) doi:10.1103/PhysRevD.94.084036 arXiv:1609.03613 [gr-qc]].

[36] B. Koch, I. A. Reyes and Á. Rincón, Class. Quant. Grav. 33, no. 22, 225010 (2016) doi:10.1088/02649381/33/22/225010 arXiv:1606.04123 [hep-th]].

[37] A. Platania and F. Saueressig, Found. Phys. 48, no. 10,1291 (2018) doi:10.1007/s10701-018-0181-0 arXiv:1710.01972 [hep-th]].

[38] A. Bonanno, S. J. Gabriele Gionti and A. Platania, Class. Quant. Grav. 35, no. 6, 065004 (2018) doi:10.1088/1361-6382/aaa535 arXiv:1710.06317 [grqc]].

[39] Á. Rincón, B. Koch and I. Reyes, J. Phys. Conf. Ser. 831, no. 1, 012007 (2017) doi:10.1088/17426596/831/1/012007 arXiv:1701.04531 [hep-th]].

[40] Á. Rincón, E. Contreras, P. Bargueño, B. Koch, G. Panotopoulos and A. Hernández-Arboleda, Eur. Phys. J. C 77, no. 7, 494 (2017) doi:10.1140/epjc/s10052-017-50459 arXiv:1704.04845 [hep-th]].

[41] L. Modesto, L. Rachwal and I. L. Shapiro, Eur. Phys. J. C 78, no. 7, 555 (2018) doi:10.1140/epjc/s10052-0186035-2 arXiv:1704.03988 [hep-th]].

[42] E. Contreras, Á. Rincón, B. Koch and P. Bargueño, Int. J. Mod. Phys. D 27, no. 03, 1850032 (2017) doi:10.1142/S0218271818500323 arXiv:1711.08400 [gr$\mathrm{qc}]]$.

[43] X. Calmet, S. Capozziello and D. Pryer, Eur. Phys. J. C 77, no. 9, 589 (2017) doi:10.1140/epjc/s10052-0175172-3 arXiv:1708.08253 [hep-th]].

[44] J. D. Toniato, D. C. Rodrigues, Á. O. F. de Almeida and N. Bertini, Phys. Rev. D 96, no. 6, $064034 \quad$ (2017) doi:10.1103/PhysRevD.96.064034 arXiv:1706.09032 [gr-qc]].

[45] B. S. Merzlikin, I. L. Shapiro, A. Wipf and O. Zanusso, Phys. Rev. D 96, no. 12, 125007 (2017) doi:10.1103/PhysRevD.96.125007 arXiv:1711.02224 [hep-th]].

[46] Á. Rincón and G. Panotopoulos, Phys. Rev. D 97, no. 2, 024027 (2018) doi:10.1103/PhysRevD.97.024027 arXiv:1801.03248 [hep-th]].

[47] A. Hernández-Arboleda, Á. Rincón, B. Koch, E. Contreras and P. Bargueño, arXiv:1802.05288 [gr-qc].

[48] E. Contreras, Á. Rincón, B. Koch and P. Bargueño, Eur. Phys. J. C 78, no. 3, 246 (2018) doi:10.1140/epjc/s10052-018-5709-0 arXiv:1803.03255 [gr-qc]].

[49] Á. Rincón and B. Koch, Eur. Phys. J. C 78, no. $12, \quad 1022$ (2018) doi:10.1140/epjc/s10052-018-6488-3 arXiv:1806.03024 [hep-th]].

[50] Á. Rincón, E. Contreras, P. Bargueño, B. Koch and G. Panotopoulos, Eur. Phys. J. C 78, no. 8, 641 (2018) doi:10.1140/epjc/s10052-018-6106-4 arXiv:1807.08047 [hep-th]].

[51] X. Calmet, Phys. Lett. B 787, $36 \quad$ (2018) doi:10.1016/j.physletb.2018.10.040 arXiv:1810.09719 [hep-th]].

[52] E. Contreras, Á. Rincón and J. M. RamírezVelasquez, Eur. Phys. J. C 79, no. 1, 53 (2019) doi:10.1140/epjc/s10052-019-6601-2 arXiv:1810.07356 [gr-qc]].

[53] A. Bonanno, A. Platania and F. Saueressig, Phys. Lett. B 784, 229 (2018) doi:10.1016/j.physletb.2018.06.047 arXiv:1803.02355 [gr-qc]]. 
[54] E. Contreras and P. Bargueño, Mod. Phys. Lett. A 33, no. 32, 1850184 (2018) doi:10.1142/S0217732318501845 arXiv:1809.00785 [gr-qc]].

[55] E. Contreras and P. Bargueño, Int. J. Mod. Phys. D 27, no. 09, 1850101 (2018) doi:10.1142/S0218271818501018 arXiv:1804.00988 [gr-qc]].

[56] V. Sahni, Class. Quant. Grav. 19, 3435 (2002) doi:10.1088/0264-9381/19/13/304 astro-ph/0202076.

[57] T. Prokopec, gr-qc/0603088.

[58] S. J. Brodsky and R. Shrock, Proc. Nat. Acad. Sci. 108, 45 (2011) doi:10.1073/pnas.1010113107 arXiv:0905.1151 [hep-th]].

[59] N. Kaloper and A. Padilla, Phys. Rev. Lett. 112, no. 9, 091304 (2014) doi:10.1103/PhysRevLett.112.091304 arXiv:1309.6562 [hep-th]].

[60] D. Stojkovic, Mod. Phys. Lett. A 28, 1330034 (2013) doi:10.1142/S0217732313300346 arXiv:1406.2696 [grqc]].

[61] A. Padilla, arXiv:1502.05296 [hep-th].

[62] S. D. Bass, Mod. Phys. Lett. A 30, no. 22, $1540033 \quad$ (2015) doi:10.1142/S0217732315400337 arXiv:1503.05483 [hep-ph]].

[63] E. A. Novikov, Mod. Phys. Lett. A 31, no. 15, 1650092 (2016). doi:10.1142/S0217732316500929

[64] S. Nojiri, Mod. Phys. Lett. A 31, no. 37, 1650213 (2016) doi:10.1142/S0217732316502138 arXiv:1601.02203 [hep-th]].

[65] J. Biemans, A. Platania and F. Saueressig, Phys. Rev. D 95, no. 8, $086013 \quad$ (2017) doi:10.1103/PhysRevD.95.086013 arXiv:1609.04813 [hep-th]].

[66] S. Hossenfelder, arXiv:1801.02176 [physics.hist-ph].

[67] S. Weinberg, doi:10.1007/978-1-4684-0931-4 1

[68] S. W. Hawking and W. Israel,

[69] C. Wetterich, Phys. Lett. B 301, 90 (1993) doi:10.1016/0370-2693(93)90726-X arXiv:1710.05815 [hep-th]].

[70] T. R. Morris, Int. J. Mod. Phys. A 9, 2411 (1994) doi:10.1142/S0217751X94000972 hep-ph/9308265.

[71] A. Bonanno and M. Reuter, Phys. Rev. D 62, 043008 (2000) doi:10.1103/PhysRevD.62.043008 hepth/0002196.

[72] D. F. Litim and J. M. Pawlowski, Phys. Rev. D 66, 025030 (2002) doi:10.1103/PhysRevD.66.025030 hepth/0202188.

[73] M. Reuter and H. Weyer, Phys. Rev. D 70, 124028 (2004) doi:10.1103/PhysRevD.70.124028 hepth/0410117.

[74] A. Bonanno and M. Reuter, Phys. Rev. D 73, 083005 (2006) doi:10.1103/PhysRevD.73.083005 hepth/0602159.

[75] M. Niedermaier, Class. Quant. Grav. 24, R171 (2007) doi:10.1088/0264-9381/24/18/R01 gr-qc/0610018.

[76] R. Percacci, In *Oriti, D. (ed.): Approaches to quantum gravity* 111-128 arXiv:0709.3851 [hep-th]].

[77] A. Bonanno and M. Reuter, Phys. Rev. D 60, 084011 (1999) doi:10.1103/PhysRevD.60.084011 grqc/9811026.

[78] H. Emoto, hep-th/0511075

[79] M. Reuter and E. Tuiran, doi:10.1142/9789812834300 0473 hep-th/0612037

[80] B. F. L. Ward, Acta Phys. Polon. B 37, 1967 (2006) hep-ph/0605054.

[81] B. Koch, Phys. Lett. B 663, 334 (2008) doi:10.1016/j.physletb.2008.04.025

[hep-ph]].

[82] J. Hewett and T. Rizzo, JHEP 0712, 009 (2007) doi:10.1088/1126-6708/2007/12/009 arXiv:0707.3182 [hep-ph]].

[83] D. F. Litim and T. Plehn, Phys. Rev. Lett. 100, 131301 (2008) doi:10.1103/PhysRevLett.100.131301 arXiv:0707.3983 [hep-ph]].

[84] T. Burschil and B. Koch, Zh. Eksp. Teor. Fiz. 92, 219 (2010) [JETP Lett. 92, 193 (2010)] doi:10.1134/S0021364010160010 arXiv:0912.4517 [hep$\mathrm{ph}]$.

[85] K. Falls, D. F. Litim and A. Raghuraman, Int. J. Mod. Phys. A 27, 1250019 (2012) doi:10.1142/S0217751X12500194 arXiv:1002.0260 [hep-th]].

[86] R. Casadio, S. D. H. Hsu and B. Mirza, Phys. Lett. B 695, 317 (2011) doi:10.1016/j.physletb.2010.10.060 arXiv:1008.2768 [gr-qc]].

[87] S. Basu and D. Mattingly, Phys. Rev. D 82 , $124017 \quad$ (2010) doi:10.1103/PhysRevD.82.124017 arXiv:1006.0718 [hep-th]].

[88] M. Reuter and E. Tuiran, Phys. Rev. D 83, $044041 \quad$ (2011) doi:10.1103/PhysRevD.83.044041 arXiv:1009.3528 [hep-th]].

[89] Y. F. Cai and D. A. Easson, JCAP 1009, 002 (2010) doi:10.1088/1475-7516/2010/09/002 arXiv:1007.1317 [hep-th]].

[90] K. Falls and D. F. Litim, Phys. Rev. D 89, $084002 \quad$ (2014) doi:10.1103/PhysRevD.89.084002 arXiv:1212.1821 [gr-qc]].

[91] D. Becker and M. Reuter, JHEP 1207, 172 (2012) doi:10.1007/JHEP07(2012)172 arXiv:1205.3583 [hepth].

[92] D. Becker and M. Reuter, doi:10.1142/9789814623995 0405 arXiv:1212.4274 [hep-th].

[93] B. Koch and F. Saueressig, Class. Quant. Grav. 31, 015006 (2014) doi:10.1088/0264-9381/31/1/015006 arXiv:1306.1546 [hep-th]].

[94] C. González and B. Koch, Int. J. Mod. Phys. A 31, no. 26, 1650141 (2016) doi:10.1142/S0217751X16501414 arXiv:1508.01502 [hep-th]].

[95] R. Torres, Phys. Rev. D 95, no. 12, 124004 (2017) doi:10.1103/PhysRevD.95.124004 arXiv:1703.09997 [gr-qc]].

[96] B. Knorr and S. Lippoldt, Phys. Rev. D 96, no. 6, 065020 (2017) doi:10.1103/PhysRevD.96.065020 arXiv:1707.01397 [hep-th]].

[97] A. Eichhorn, Found. Phys. 48, no. 10, 1407 (2018) doi:10.1007/s10701-018-0196-6 arXiv:1709.03696 [grqc]].

[98] J. M. Pawlowski and D. Stock, Phys. Rev. D 98, no. 10, 106008 (2018) doi:10.1103/PhysRevD.98.106008 arXiv:1807.10512 [hep-th]].

[99] N. Alkofer, arXiv:1810.03132 [hep-th].

[100] A. Eichhorn, arXiv:1810.07615 [hep-th].

[101] B. Koch and I. Ramirez, Class. Quant. Grav. 28, 055008 (2011) doi:10.1088/0264-9381/28/5/055008 arXiv:1010.2799 [gr-qc]].

[102] S. Domazet and H. Stefancic, Class. Quant. Grav. 29, 235005 (2012) doi:10.1088/0264-9381/29/23/235005 arXiv:1204.1483 [gr-qc]].

[103] B. Koch, P. Rioseco and C. Contreras, Phys. Rev. D 91, no. 2, 025009 (2015) doi:10.1103/PhysRevD.91.025009 
arXiv:1409.4443 [hep-th]]

[104] E. Manrique and M. Reuter, Annals Phys. 325, 785 (2010) doi:10.1016/j.aop.2009.11.009 arXiv:0907.2617 [gr-qc]].

[105] E. Manrique, M. Reuter and F. Saueressig, Annals Phys. 326, 463 (2011) doi:10.1016/j.aop.2010.11.006 arXiv:1006.0099 [hep-th]].

[106] E. Manrique, M. Reuter and F. Saueressig, Annals Phys. 326, 440 (2011) doi:10.1016/j.aop.2010.11.003 arXiv:1003.5129 [hep-th]].

[107] D. Becker and M. Reuter, Annals Phys. 350, 225 (2014) doi:10.1016/j.aop.2014.07.023 arXiv:1404.4537 [hep-th]].

[108] J. A. Dietz and T. R. Morris, JHEP 1504, 118 (2015) doi:10.1007/JHEP04(2015)118 arXiv:1502.07396 [hepth]].

[109] P. Labus, T. R. Morris and Z. H. Slade, Phys. Rev. D 94, no. 2, 024007 (2016) doi:10.1103/PhysRevD.94.024007 arXiv:1603.04772 [hep-th]].

[110] T. R. Morris, JHEP 1611, 160 (2016) doi:10.1007/JHEP11(2016)160 arXiv:1610.03081 [hep-th]].

[111] N. Ohta, PTEP 2017, no. 3, 033E02 (2017) doi:10.1093/ptep/ptx020 arXiv:1701.01506 [hep-th]].

[112] B. Koch and F. Saueressig, Int. J. Mod. Phys. A 29, no. 8, 1430011 (2014) doi:10.1142/S0217751X14300117 arXiv:1401.4452 [hep-th]].

[113] Á. Rincón and B. Koch, J. Phys. Conf. Ser. 1043, no. 1, 012015 (2018) doi:10.1088/1742-6596/1043/1/012015 arXiv:1705.02729 [hep-th]].

[114] T. Jacobson, Class. Quant. Grav. 24, 5717 (2007) doi:10.1088/0264-9381/24/22/N02 arXiv:0707.3222 [gr-qc]].

[115] M. Visser and C. Barcelo, doi:10.1142/9789812792129 0014 gr-qc/0001099

[116] F. Canales, B. Koch, C. Laporte and Á. Rincón, arXiv:1812.10526 [gr-qc].

[117] C. Patrignani et al. [Particle Data Group], Chin. Phys. C 40, no. 10, 100001 (2016). doi:10.1088/1674$1137 / 40 / 10 / 100001$

[118] T. Banks and W. Fischler, astro-ph/0307459.

[119] E. W. Kolb and M. S. Turner, Front. Phys. 69, 1 (1990).

[120] S. Capozziello, R. de Ritis and A. A. Marino, Nuovo Cim. B 112, 1351 (1997) astro-ph/9605176.

[121] X. Calmet and H. Fritzsch, Eur. Phys. J. C
24, 639 (2002) doi:10.1007/s10052-002-0976-0 hep$\mathrm{ph} / 0112110$.

[122] S. Carneiro, doi:10.1142/9789812704030 0204 grqc/0307114

[123] A. Reisenegger, P. Jofré and R. Fernández, Mem. Soc. Ast. It. 80, no. 4, 829 (2009) [Highlights Astron. 15, 314 (2010)] doi:10.1017/S1743921310009518 arXiv:0911.0190 [astro-ph.HE]].

[124] N. Yunes, F. Pretorius and D. Spergel, Phys. Rev. D 81, 064018 (2010) doi:10.1103/PhysRevD.81.064018 arXiv:0912.2724 [gr-qc]].

[125] J. P. Uzan, Living Rev. Rel. 14, 2 (2011) doi:10.12942/lrr-2011-2 arXiv:1009.5514 [astroph.CO]].

[126] H. Fritzsch, Nucl. Phys. Proc. Suppl. 203-204, 3 (2010). doi:10.1016/j.nuclphysbps.2010.08.002

[127] J. D. Anderson, G. Schubert, V. Trimble and M. R. Feldman, EPL 110, no. 1, 10002 (2015) doi:10.1209/0295-5075/110/10002 arXiv:1504.06604 [gr-qc]].

[128] L. Kantha, Adv. Astron. 2016, 9743970 (2016). doi:10.1155/2016/9743970

[129] X. Calmet, Eur. Phys. J. C 77, no. 11, 729 (2017) doi:10.1140/epjc/s10052-017-5324-5 arXiv:1707.06922 [gr-qc]].

[130] S. P. Martin, Adv. Ser. Direct. High Energy Phys. 21, 1 (2010) [Adv. Ser. Direct. High Energy Phys. 18, 1 (1998)] doi:10.1142/9789812839657 0001, 10.1142/9789814307505 0001 hep-ph/9709356.

[131] N. Arkani-Hamed, S. Dimopoulos and G. R. Dvali, Phys. Lett. B 429, 263 (1998) doi:10.1016/S03702693(98)00466-3 hep-ph/9803315.

[132] K. A. Meissner and H. Nicolai, Phys. Lett. B 648, 312 (2007) doi:10.1016/j.physletb.2007.03.023 hepth/0612165.

[133] M. Reuter, Phys. Rev. D 57, 971 (1998) doi:10.1103/PhysRevD.57.971 hep-th/9605030.

[134] M. Reuter and F. Saueressig, Phys. Rev. D 65, 065016 (2002) doi:10.1103/PhysRevD.65.065016 hepth/0110054.

[135] D. F. Litim, Phys. Rev. Lett. 92, 201301 (2004) doi:10.1103/PhysRevLett.92.201301 hep-th/0312114.

[136] With "separatrix" it is referred to the RG flow line which connects the trivial fixed point $g=\lambda=0$ with the non trivial UV fixed point $\left(g^{*}, \lambda^{*}\right)$. 